\title{
The Role of Lipid Chains as Determinants of Membrane Stability in the Presence of Styrene
}

\author{
Vivien Yeh, Alice Goode, David Johnson, Nathan Cowieson, and Boyan B. Bonev**
}

Cite This: https://doi.org/10.1021/acs.langmuir.1c02332

Read Online

ACCESS | Lill Metrics \& More | 回 Article Recommendations | si Supporting Information
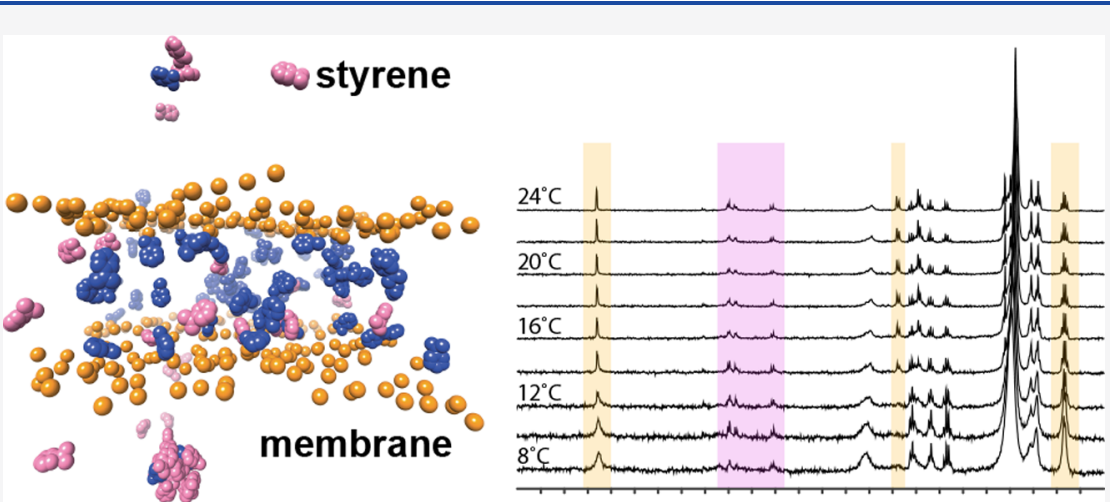

ABSTRACT: Biofermentative production of styrene from renewable carbon sources is crucially dependent on strain tolerance and viability at elevated styrene concentrations. Solvent-driven collapse of bacterial plasma membranes limits yields and is technologically restrictive. Styrene is a hydrophobic solvent that readily partitions into the membrane interior and alters membrane-chain order and packing. We investigate styrene incorporation into model membranes and the role lipid chains play as determinants of membrane stability in the presence of styrene. MD simulations reveal styrene phase separation followed by irreversible segregation into the membrane interior. Solid state NMR shows committed partitioning of styrene into the membrane interior with persistence of the bilayer phase up to $67 \mathrm{~mol} \%$ styrene. Saturated-chain lipid membranes were able to retain integrity even at 80 mol \% styrene, whereas in unsaturated lipid membranes, we observe the onset of a non-bilayer phase of small lipid aggregates in coexistence with styrene-saturated membranes. Shorter-chain saturated lipid membranes were seen to tolerate styrene better, which is consistent with observed chain length reduction in bacteria grown in the presence of small molecule solvents. Unsaturation at mid-chain position appears to reduce the membrane tolerance to styrene and conversion from cis- to trans-chain unsaturation does not alter membrane phase stability but the lipid order in trans-chains is less affected than cis.

\section{INTRODUCTION}

Polystyrene is one of the most commonly used plastics in everyday life. It is produced from monomeric styrene by suspension polymerization and is ubiquitously used in numerous important industrial polymers and co-polymer applications. Styrene is a volatile hydrophobic solvent manufactured as a commodity chemical at annual volumes of 25 million tons in a 30 billion USD market. ${ }^{1}$ Current production of styrene relies on catalytic dehydrogenation of ethylbenzene, ${ }^{1}$ which, in turn, is derived from petrochemical sources. The chemocatalytic reaction is an endothermal reaction that requires 3 tons of steam per ton of styrene produced. In 2002, ethylbenzene and styrene production were the second and third largest energy consumers in the US petrochemical industry, estimated from the production volume.

Global mandate to move manufacturing to carbon and energy neutral technologies has spurred off a rapid development of biofermentative production of commodity chemicals.
Industrial biotechnology and the use of microorganism fermentation for the production of chemicals and materials have been attracting interest, aiming for sustainable bioproduction with renewable resources, reduction in waste, and moving away from the use of petrochemical sources of carbon. $^{2,3}$

Styrene is naturally present as a minor metabolite. Several microorganisms, like Pencillium camemberti ${ }^{4}$ and members of the Styracaceae family, ${ }^{5}$ are capable of synthesizing low levels of styrene. Natural production is extremely low and biofermentative processes using engineered bacterial hosts are presently

Received: September 1, 2021

Revised: December 16, 2021 
<smiles>C=Cc1ccccc1</smiles>

\section{Styrene}

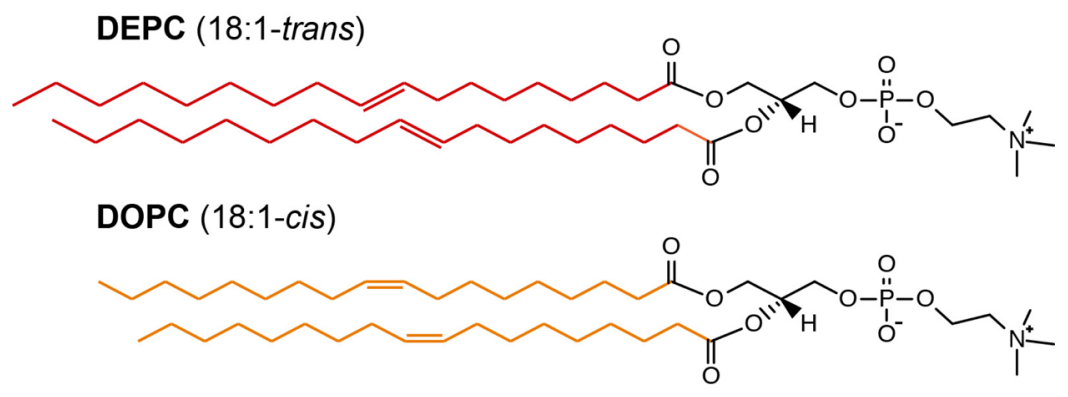

POPC (16:0, 18:1-cis)
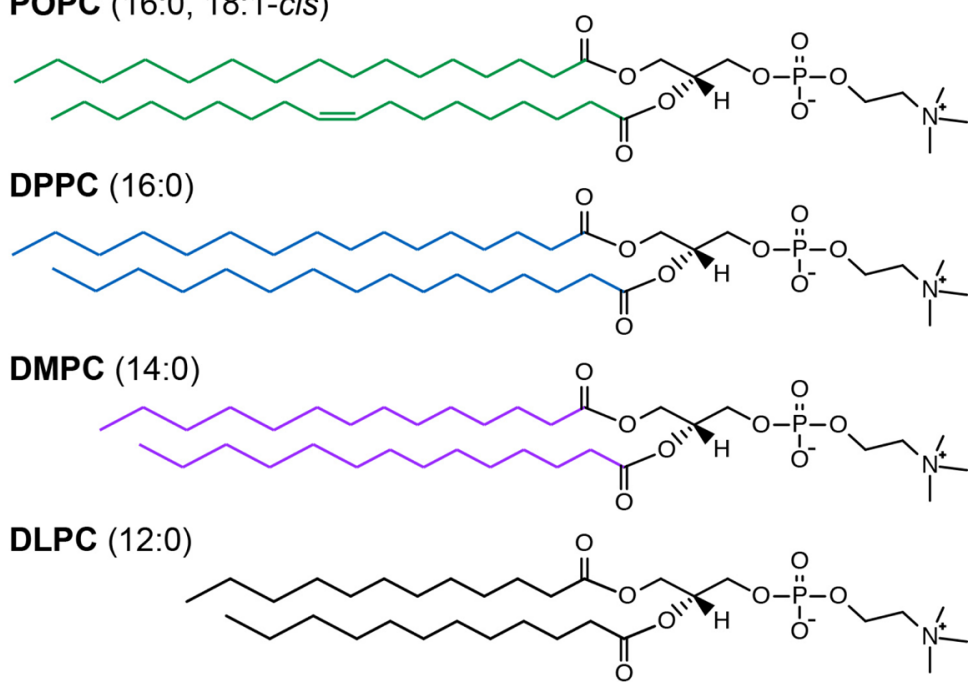

Figure 1. Chemical structures of styrene, DEPC (18:1 C9-trans), DOPC (18:1 C9-cis), POPC (16:0, 18:1 C9-cis), DPPC (16:0), DMPC (14:0), and DLPC (12:0).

developed as a sustainable approach to large-scale manufacturing.

Styrene production has been reported using engineered Escherichia coli NST74 with glucose as a carbon source and conversion of phenylalanine to cinnamate via Pal2-mediated pathway, leading to cinnamate followed by cinnamate decarboxylation and conversion to styrene by FDC1. ${ }^{6} \mathrm{~A}$ significant bottleneck of the biofermentative production is styrene toxicity to the fermentative strains, which limits production yield and strain performance. ${ }^{7,8}$ Other commodity chemicals, such as succinic or lactic acid, can be produced on the scale of $100 \mathrm{~g} / \mathrm{L}$ using E. coli chassis ${ }^{9,10}$ as soluble products. By contrast, during styrene production, its hydrophobic molecules readily partition into bacterial membranes, which leads to membrane damage and cell death. ${ }^{8}$ Membrane disruption is one of the major mechanisms of product toxicity in biofermentative production of commodity chemicals and limits yields, as well as product recovery and separation. ${ }^{11}$

In Gram-negative bacteria, the plasma membrane composition comprises $72-80 \mathrm{~mol} \%$ phsphatidylethanolamine (PE) and 21-17 mol \% negatively charged phosphatidylglycerol (PG) with 4-7 mol \% diphosphatidylglycerol (cardiolipin, CL). ${ }^{12,13}$ The phospholipid acyl chains may vary between species but can also be altered as an adaptive response to changes in growth conditions, growth phase, or other environmental factors. Industrially relevant organisms, such as E. coli and Pseudomonas putida, have been known to alter their lipid composition and adjust membrane properties in response to organic solvent-induced stress. ${ }^{14}$ Adaptive responses include shifting the ratio of saturated to unsaturated chains ${ }^{15,16}$ and cis/trans-isomerization of unsaturated fatty acid chains, ${ }^{14,17,18}$ increase in the fraction of cyclopropyl chains, ${ }^{19}$ changes in fatty acid chain length, ${ }^{19,20}$ increase in CL content, $^{19}$ changes in phospholipid headgroup distribution, ${ }^{14,21}$ and modification of LPS. ${ }^{22}$

Bacterial exposure to hydrophobic solvents can trigger adaptive changes in membrane lipid chain length, order and organization, chain packing, and membrane fluidity that affect trans-gauche isomerization and cooperative chain-chain interactions. $^{14,23}$ Such changes alter the capacity of membranes for accommodating solvent, modulate membrane transport and membrane protein function, and are important parameters in modulating membrane stability and cell viability. Solvent toxicity at high concentrations leads to changes in membrane integrity and permeability, and bilayer phase collapse into nonbilayer lipid phases, such as inverted hexagonal or micellar 
phases. Toluene, a hydrophilic aromatic compound with $\log \mathrm{P}$ of $2.5,{ }^{24}$ has been reported to disrupt severely the integrity of cell membranes. Addition of toluene results in membrane leakage that allows small molecules to permeate the membrane. ${ }^{25}$ High concentration of toluene leads to phase conversion from bilayer to inverted hexagonal phase and loss of cytoplasmic proteins, proton motive force (PMF), and, ultimately, to cell death. ${ }^{17,25}$

Membrane disruption is challenging in alcohol production, and the interaction of alcohols with lipid membranes has been studied, ${ }^{26}$ as well as the impact of alcohol on growth and lipid composition of bacteria. ${ }^{20,27,28}$ Using engineered E. coli strains, isobutanol can be produced at up to $50 \mathrm{~g} / \mathrm{L}$ in $72 \mathrm{~h}$ with in situ product removal to decrease cytotoxicity at high product levels. ${ }^{29}$ Lipid composition, in particular, lipid headgroups, was shown to have an impact on the interaction of lipids with ethanol using molecular dynamic simulations, highlighting differences in ethanol penetration and other biophysical properties such as chain order. ${ }^{30}$ We have shown that lipid chain composition is an important determinant of membrane stability in the presence of butyl methacryalte (BMA), $\log P=$ 2.88 , and that membrane tolerance to BMA incorporation depends on the saturation and isomerization of lipid hydrocarbon chains. The elongated and partially amphipathic BMA molecules intercalate into the membrane hydrophobic core and align with the lipid chains in the proximity of the backbone carbonyl groups. ${ }^{31}$

In this study, we investigate the response of model lipid membranes to the presence of styrene and the impact of lipid chain composition on membrane stability and resistance to styrene-mediated phase disruption. Such changes in lipid phase behavior can be monitored by ${ }^{31} \mathrm{P}$ wideline $\mathrm{NMR},{ }^{32,33}$ as well as by small angle $\mathrm{x}$-ray scattering (SAXS). ${ }^{34}$ Styrene has a $\log P$ of $2.95^{17}$ and readily partitions into the membrane interior. The solubility limit of styrene in water at $25^{\circ} \mathrm{C}$ is $300 \mathrm{mg} / \mathrm{L},{ }^{35}$ which is also estimated to match the in vivo styrene toxicity threshold $^{6}$ and supports a styrene toxicity model based on membrane collapse. We hypothesize that lipid chain length, saturation, and cis vs trans unsaturation affect membrane stability and can be used to engineer strains with enhanced tolerance to styrene. Membrane stability in the presence of high levels of styrene is assessed in membranes of same headgroup lipid, phosphatidylcholine (PC), but with a different lipid acyl chain length, saturation, and cis-trans isomerization. Lipids with PC headgroups are used to study styrene incorporation into the membrane hydrophobic interior as the PC headgroups are comparatively nonreactive and provide a stable bilayer platform for systematic investigation of changes in bilayer phase state, organization, and dynamics in response to variation in lipid chain composition.

Here, we use MD simulations to model the molecular process of styrene partitioning into lipid membranes and use a combination of solid state NMR and SAXS to characterize the role of lipid chain composition on membrane stability in the presence of styrene. Saturated chain length was varied from 12 to 16 carbons (DLPC, DMPC and DPPC) and compared to 18-carbon, mid-chain cis-unsaturated DOPC or trans-unsaturated DEPC, as well as a common mixed-chain POPC. Wideline ${ }^{31} \mathrm{P}$ solid state NMR was used to monitor phase stability and collapse, and SAXS to monitor changes in membrane thickness and phase changes. Styrene incorporation and interactions with the membrane interior are monitored by high-resolution ${ }^{13} \mathrm{C}$ solid state $\mathrm{NMR}$, which also informs on changes in internal lipid chain order and dynamics, main transition temperature, and lipid segmental mobility.

\section{EXPERIMENTAL METHODS}

Synthetic lipids, DOPC (1,2-dioleoyl-sn-glycero-3-phosphocholine), DMPC (1,2-dimyristoyl-sn-glycero-3-phosphocholine), DLPC (1,2dilauroyl-sn-glycero-3-phosphocholine), DPPC (1,2-dipalmitoyl-snglycero-3-phosphocholine), POPC (1-palmitoyl-2-oleoyl-glycero-3phosphocholine), and DEPC (1,2-dielaidoyl-sn-glycero-3-phosphocholine) were purchased from Avanti Polar Lipids (AL, USA) at $>98 \%$ purity and used without further purification. Styrene was purchased from Sigma-Aldrich at $>99 \%$ purity and used as supplied. The structure of the lipids and styrene is shown in Figure 1.

Preparation of Multilamellar Vesicles. Styrene was added at the desired molar ratio to $20 \mu \mathrm{mol}$ of lipid powder and mixed for 5 min with a fine glass rod. The sample was then suspended in $500 \mu \mathrm{L}$ of HPLC-grade water and stirred for a further $5 \mathrm{~min}$, before another $500 \mu \mathrm{L}$ of water were added. The sample was mixed and freezethawed five times between -196 and $50{ }^{\circ} \mathrm{C}$ and stored at $4{ }^{\circ} \mathrm{C}$ overnight. Multilamellar vesicles (MLV) obtained in this way were centrifuged at $16,060 \times g$ (Biofuge pico) for $20 \mathrm{~min}$ at room temperature. After the supernatant was removed, the hydrated pellet was loaded into a $4 \mathrm{~mm} \mathrm{ZrO}_{2}$ MAS NMR rotor.

Solid State NMR. All solid state NMR experiments were performed on a Varian $400 \mathrm{MHz}$ VNMRS spectrometer equipped with a $4 \mathrm{~mm}$ MAS NMR probe. Temperature was regulated using balanced heated/vortex tube-cooled gas flow. ${ }^{36,37}$ All ${ }^{31} \mathrm{P}$ spectra were referenced externally to $0 \mathrm{ppm}$ against 10 vol $\% \mathrm{H}_{3} \mathrm{PO}_{4}$ and ${ }^{13} \mathrm{C}$ spectra were referenced externally to adamantine $\mathrm{CH}_{2}$ at $37.54 \mathrm{ppm}$. Phosphorus-31 wideline NMR was carried out at a frequency of $161.82 \mathrm{MHz}$ and at a temperature of either $20^{\circ} \mathrm{C}$ for DOPC, POPC, and DEPC; $28{ }^{\circ} \mathrm{C}$ for DMPC; or $42{ }^{\circ} \mathrm{C}$ for DPPC using Hahn echo with $100 \mathrm{kHz} \pi / 2$ and $\pi$-pulses separated by $12 \mu$ s interpulse and preacquisition delays. Spectra were acquired with $25 \mathrm{~ms}$ acquisition time with a recycle delay of $5 \mathrm{~s}$ and with 1024 transients averaged to obtain each FID. All wideline spectra were processed with $100 \mathrm{~Hz}$ Lorentzian line broadening. High-resolution ${ }^{13} \mathrm{C}$ CP MAS NMR was done at a MAS frequency of $5 \mathrm{kHz}$. The $120 \mathrm{kHz}{ }^{1} \mathrm{H}$ excitation pulse was followed by $3.5 \mathrm{~ms}$ of $45 \mathrm{kHz}$ Harmann-Hahn contact for magnetization transfer to ${ }^{13} \mathrm{C}$. Spectra were acquired under $60 \mathrm{kHz}$ SPINAL- $64^{38}$ decoupling over $125 \mathrm{~ms}$ acquisition time and by averaging 1024 transients per FID or under $109 \mathrm{kHz}$ frequency switched Lee-Goldburg (FSLG) decoupling ${ }^{39}$ over $75 \mathrm{~ms}$ acquisition time by averaging 8192 transients, respectively. The recycle delay was set to $3.5 \mathrm{~s}$ to exceed five times proton $T_{1}(\sim 0.5 \mathrm{~s})$ and all spectra were processed with $2 \mathrm{~Hz}$ Lorentzian line broadening.

Small Angle X-ray Scattering. All SAXS experiments were performed on the Diamond Light Source bending beamline B21. Samples were loaded on a 96-well plate and delivered to the X-ray beam using an Arinax BioSAXS automated liquid handling robot. The samples of hydrated MLV were prepared and subjected to five freezethawing cycles on the day of the experiment. The X-ray sample environment consisted of a $1.6 \mathrm{~mm}$ diameter, $10 \mu \mathrm{m}$ thick quartz capillary held in vacuum. The experiments were done at temperature above the main transition temperature of each lipid: DOPC and $\mathrm{DEPC}$ at $20{ }^{\circ} \mathrm{C}$, DMPC at $30{ }^{\circ} \mathrm{C}$, and DPPC at $45{ }^{\circ} \mathrm{C}$. The experimental procedure consisted of a detergent wash followed by water rinse and air-dry cycle between each measurement. With each measurement, $30 \mu \mathrm{L}$ of sample was aspirated into the capillary and advanced as a series of $1 \mathrm{~s}$ frames to a total of $30 \mathrm{~s}$ was collected with a vacuum Eiger $4 \mathrm{M}$ detector. The X-ray beam measured $800 \mu \mathrm{m}^{2}$ at a photon flux of $2 \times 10^{12}$ photons per second with the detector at $2.4 \mathrm{~m}$ from the sample position yielding a scattering vector, $q$-range of 0.012 $\AA^{-1}<0.35 \AA^{-1}$, where $q$ is $4 \pi \times \sin \theta / \lambda, 2 \theta$ is the scattering angle, and $\lambda$ is the wavelength in $\AA$. To prevent sedimentation of MLVs prior to measurement, the sample was refluxed before aspiration into the measurement capillary for X-ray detection. Two independent measurements were performed using separate sample preparations on different beamtime sessions, and the variance in first-order Bragg 
reflection $\left(q \approx 0.1 \AA^{-1}\right)$ was $0.0001 \AA^{-1}$. The associated relative uncertainty $\Delta q / q \approx 0.001$ has been used to determine the $0.1 \AA$ accuracy in calculated $d$-values. In line with accepted practice, all $q$ values and $d$-spacings are reported to that accuracy without further explicit statement of this error margin.

Molecular Dynamics Simulations. Both equilibration and production MD simulations were done on a Supermicro server with multiple NVIDIA K80 and K40 general purpose graphical processing units, GPGPUs, using CHARMM $^{40}$ scripts and NAMD 2.12. Bilayer assembly and hydration in aqueous buffer with styrene were done in CHARMM-GUI ${ }^{42}$ using the Multicomponent Builder module. A $100 \times 100 \AA$ DOPC membrane patch was assembled and hydrated in $150 \mathrm{mM} \mathrm{KCl}$ with 100 styrene molecules, all positioned initially in the aqueous phase. The ensemble was equilibrated at a constant volume, temperature, and particle number (NPT) at $1 \mathrm{fs}$ steps for $750 \mathrm{ps}$ and at a constant pressure (NPT) with $2 \mathrm{fs}$ increments for a further $750 \mathrm{ps}$. Production runs were carried out in blocks of $100 \mathrm{~ns}$ at $2 \mathrm{fs}$ increments under Langevin pistoncontrolled isobaric conditions (NPT) at $30{ }^{\circ} \mathrm{C}$. Molecular visualization and trajectory analysis were done in UCSF Chimera. ${ }^{43}$

\section{RESULTS}

Biofermentative production of styrene takes place in the bacterial cytosol and bacterial plasma membranes are directly exposed to high styrene concentrations. To understand the interaction between styrene and hydrated bilayer membranes, we performed all-atom MD simulations, in which a $100 \times 100$ $\AA$ DOPC bilayer patch was hydrated in aqueous phase containing 100 styrene molecules. Styrene has apolar molecules that show strong preference for partitioning into aliphatic environments, such as the lipid membrane interior. Styrene diffusion within the aqueous subphase is rapid, and we observed styrene penetration into the membrane interior during the quick $10 \mathrm{~ns}$ preproduction runs. Frames from the $300 \mathrm{~ns}$ evolution trajectory at $1,46,100$, and $300 \mathrm{~ns}$ are shown in Figure 2. Styrene hydrophobicity drives a rapid phase

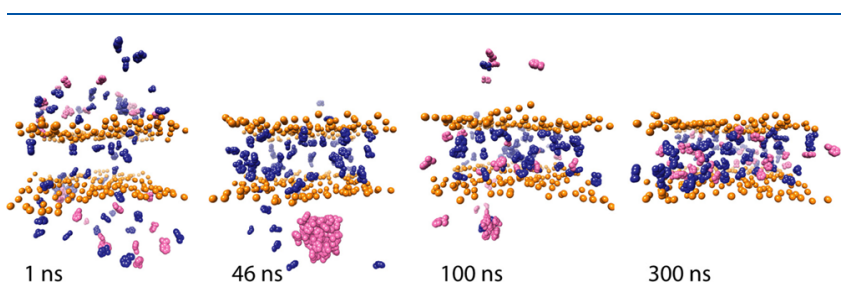

Figure 2. Frames from a $300 \mathrm{~ns}$ evolution trajectory in an all-atom MD simulation of a $100 \times 100 \AA$ membrane patch of DOPC hydrated in aqueous subphase containing 100 styrene molecules (blue). The lipid phosphorus atoms are shown in orange to mark the polar/apolar interfaces of the membrane. Frames at 1, 46, 100, and 300 ns show rapid styrene diffusion into the bilayer. Styrene concentration gradient allows a styrene phase to form away from the membrane patch (pink), which is in rapid exchange with styrene monomers in solution. By 300 ns, styrene partitions irreversibly into the membrane.

separation, observed as the pink droplet at $46 \mathrm{~ns}$. This occurs away from the membrane, as styrene molecules in the vicinity of the DOPC membrane patch are segregated irreversibly into the hydrophobic chain interior, leading to localized depletion near the membrane surface. The styrene droplets are in rapid exchange with monomeric styrene in solution, and we observe mixed styrene populations within the droplet (pink and blue molecules) throughout the first $100 \mathrm{~ns}$ of evolution. Eventually, all styrene molecules are segregated irreversibly into the hydrophobic membrane interior by the end of the $300 \mathrm{~ns}$ simulation. The final styrene/DOPC ratio is approximately 1:1.5.

Previous study showed that styrene tolerance of E. coli can be improved by membrane engineering. E. coli constructed to produce non-native trans unsaturated fatty acids by expression of cis-trans isomerase ( $\mathrm{Cti}$ ) from $P$. aeruginosa showed improved tolerance to various biorenewable products such as alcohol, organics acids, and aromatic compounds, including styrene. ${ }^{44}$ To understand membrane stability in the presence of styrene, we use wideline solid state ${ }^{31} \mathrm{P}$ NMR in combination with SAXS. Phosphorus-31 wideline NMR is very sensitive to changes in the lipid phase behavior, detected through changes in the dynamics of lipid headgroup phosphates. In addition, periodic lipid architectures, such as multilamellar repeats or inverted hexagonal phases, $\mathrm{H}_{\mathrm{I}}$, are identifiable through unique Bragg reflection patterns observed by SAXS.

Wideline ${ }^{31} \mathrm{P}$ NMR spectra are dominated by the motionally averaged effective CSA of lipid phosphates and in fluid lipid bilayers this results in Pake patterns, ${ }^{45}$ reflecting the spherically symmetric orientational distribution of lipid molecules undergoing fast axial rotation. The effective ${ }^{31} \mathrm{P}$ CSA of membrane lipids in the liquid crystalline phase is around $40-45 \mathrm{ppm}$ and can be modulated further by membrane undulations and angular molecular excursions, ${ }^{46}$ cholesterol content, ${ }^{47}$ and degree of hydration. ${ }^{48}$ Small molecular assemblies, such as aggregates, small unilamellar vesicles, or micelles, undergo rapid tumbling that leads to collapse of the orientationdependent interactions and give rise to a sharp ${ }^{31} \mathrm{P}$ NMR peak at the isotropic chemical shift of the relevant phosphate. ${ }^{49}$

Charge neutral PC liposomes were chosen for this study as they provide stable bilayer structures in the absence of membrane proteins. Although the use of PE/PG lipid mixtures may represent better the native lipid composition of $E$. coli, unsupported PE/PG membranes are intrinsically unstable, as PE favors negative bilayer curvature in balance with charge repulsion in PG. To focus on chain-specific differences in membrane stability in response to styrene, phase-stable PC bilayers with different acyl chain structure were used. Of particular interest are symmetric chain lipids with cisunsaturated $\mathrm{C} 18: 1 \Delta 9$ chains (DOPC) or mixed-chain lipids with saturated C16:0 and cis-unsaturated chains (POPC), in which the hydrophobic thickness of the cis-unsaturated oleic chain is matched to the saturated palmitic length. These model well the chain composition of inner bacterial membrane lipids, as well as the inner leaflet of the outer membranes, whereas saturated C14:0 chain lipids match the chain composition of the lipopolysaccharide outer membrane exterior.

We investigate the effects of styrene concentration on the stability of cis-unsaturated and saturated chain bilayers using ${ }^{31} \mathrm{P}$ wideline NMR. Styrene was added at $1: 2,1: 1,2: 1$, and $3: 1$ styrene/lipid molar ratios to DOPC, C18:1 $\Delta 9$-cis, and the ${ }^{31} \mathrm{P}$ NMR spectra from hydrated membranes are shown in Figure 3a. The corresponding spectra from styrene in saturated C14:0, DMPC, membranes are shown in Figure $3 \mathrm{~b}$. The experiments were recorded above the lipid main transition temperatures of the respective lipid, which occur at $-17^{\circ} \mathrm{C}$ for DOPC ${ }^{50}$ and $24^{\circ} \mathrm{C}$ for DMPC. ${ }^{51}$ All spectra are dominated by Pake distributions of approximately $45 \mathrm{ppm}$ width, revealing axially symmetric lipid motions and the presence of hydrated bilayers in the liquid crystalline phase.

Unsaturated DOPC membranes show high tolerance to styrene incorporation and retained bilayer stability up to 67 mol \% styrene (2,1 styrene/DOPC molar ratio), seen in Pake 


\section{(a) DOPC}

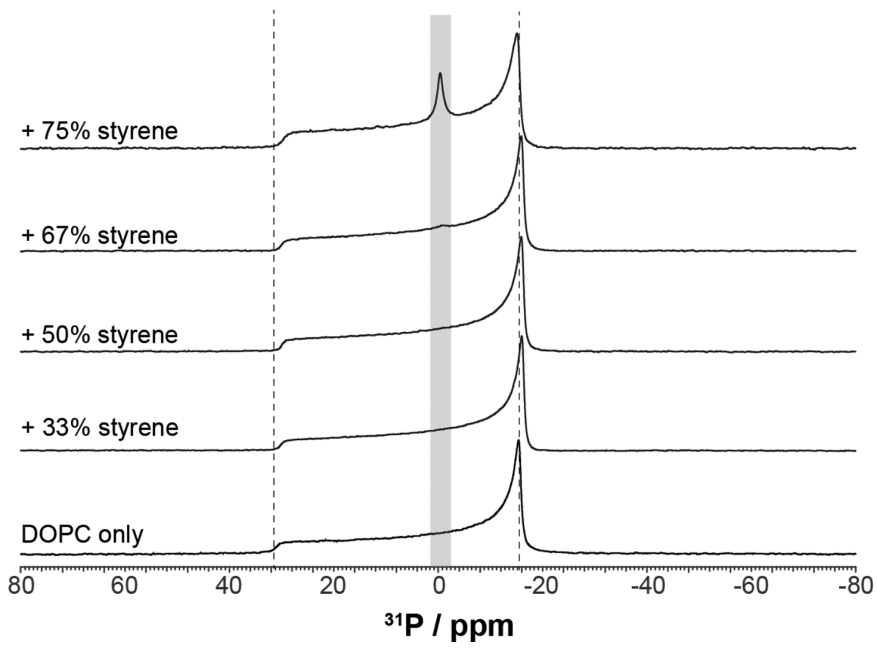

(b) DMPC

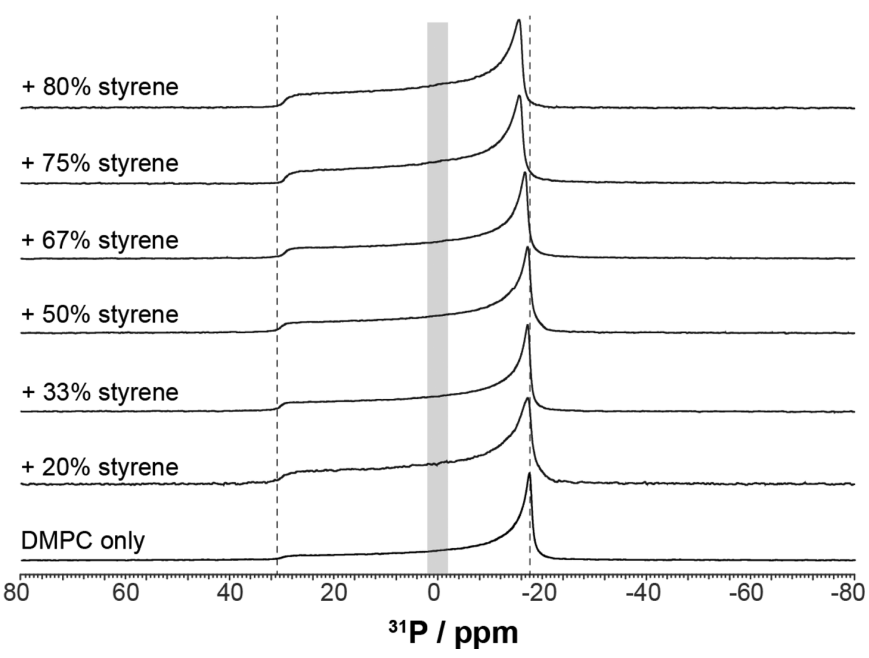

Figure 3. Wideline ${ }^{31} \mathrm{P}$ NMR spectra of hydrated (a) DOPC (18:1 c9-cis) MLV alone and with 33, 50, 67, and 75 mol \% of styrene, acquired at 20 ${ }^{\circ} \mathrm{C}$; and (b) DMPC (14:0) MLV alone and with 20, 33, 50, 67, 75, and $80 \mathrm{~mol} \%$ of styrene acquired at $28{ }^{\circ} \mathrm{C}$, above $T_{\mathrm{m}}=24{ }^{\circ} \mathrm{C}$ for DMPC. Gray shading highlights isotropic peak associated with non-bilayer phase onset and membrane disruption, characteristic of membrane disruption at DOPC with $75 \%$ styrene. Dashed lines guide the eye to the 0 and $90^{\circ}$ edges of the Pake pattern to illustrate slight reduction in effective CSA and subtle increase in molecular disorder of DMPC with increasing styrene concentration above $67 \mathrm{~mol} \%$.

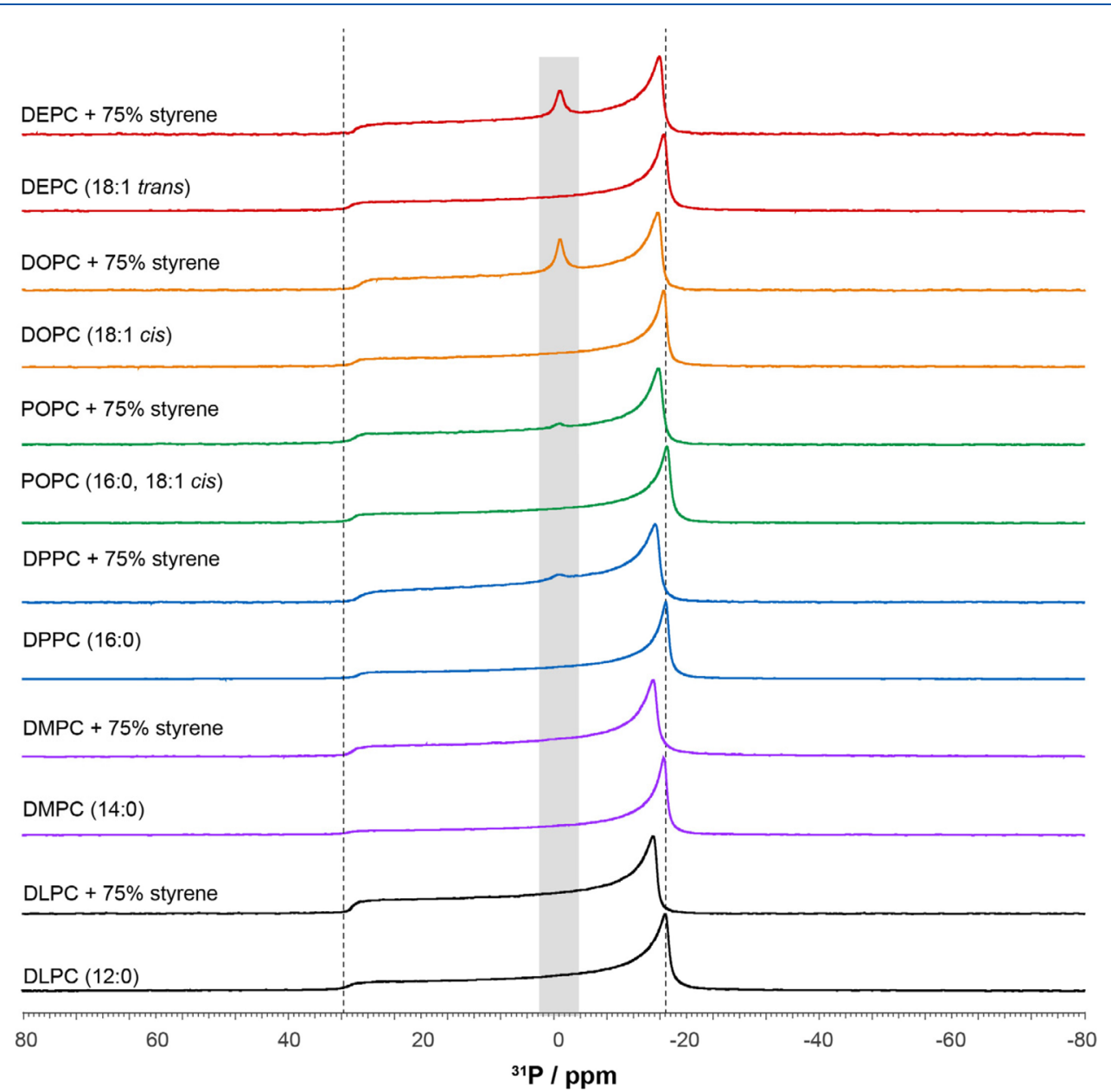

Figure 4. Wideline ${ }^{31} \mathrm{P}$ NMR spectra of hydrated MLVs composed of (red) DEPC, (orange) DOPC, (green) POPC, (blue) DPPC, (purple) DMPC, and (black) DLPC, containing either (upper) no styrene and (lower) $75 \mathrm{~mol} \%$ of styrene. The spectra were recorded in the fluid, $\mathrm{L}_{\alpha}$ phase at $20^{\circ} \mathrm{C}$ for DEPC, DOPC, POPC, and DLPC, at $28{ }^{\circ} \mathrm{C}$ for DMPC, and $42{ }^{\circ} \mathrm{C}$ for DPPC membranes. Gray shading highlights expected isotropic contribution from non-bilayer lipid environment coexisting with the styrene-saturated membrane; dotted lines show the variation in Pake pattern width and effective CSA after addition of styrene to $75 \mathrm{~mol} \%$. 


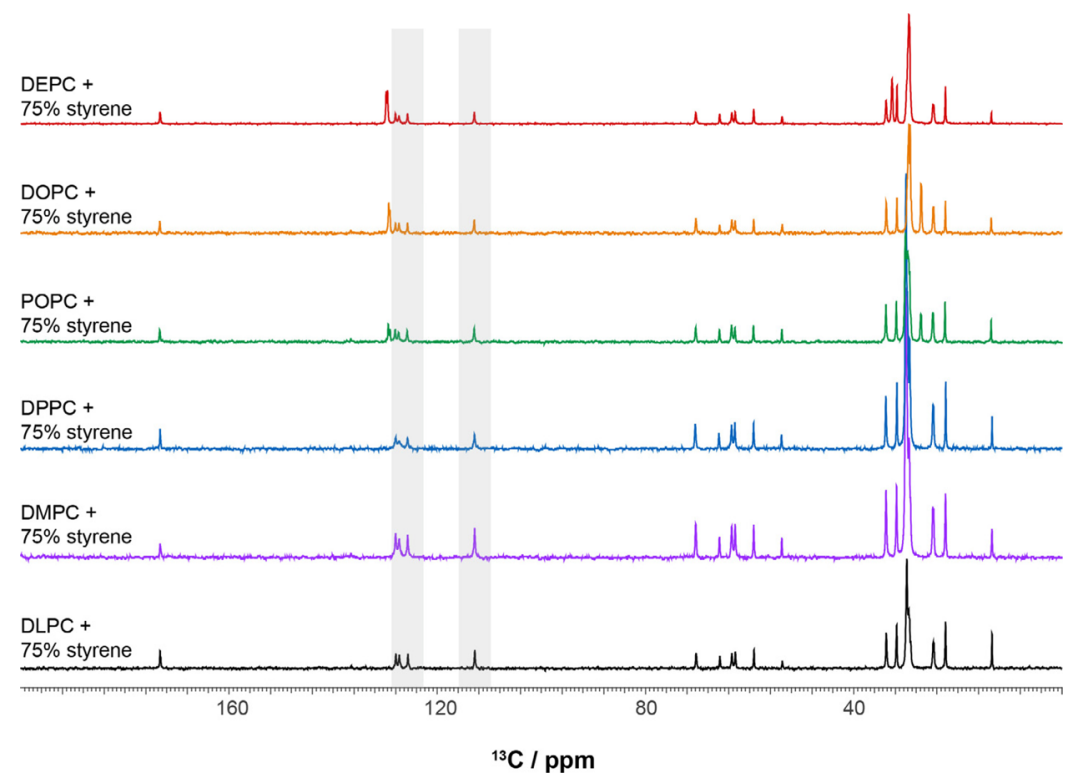

Figure 5. ${ }^{13} \mathrm{C}$ MAS CP NMR spectra of (red) DEPC, (orange) DOPC, (green) POPC, (blue) DPPC, (purple) DMPC, and (black) DLPC with $75 \%$ styrene spinning at $5 \mathrm{kHz}$, recorded using SPINAL decoupling. The spectra were recorded in the fluid $\mathbf{L}_{\alpha}$ phase at $24^{\circ} \mathrm{C}$ for DEPC, DOPC, POPC, and DLPC; $32{ }^{\circ} \mathrm{C}$ for DMPC; and $46^{\circ} \mathrm{C}$ for DPPC samples. The gray panels highlight styrene resonances observed in the CP spectra.

distribution-dominated ${ }^{31} \mathrm{P}$ wideline spectra (Figure 3a). At a higher concentration of styrene $(75 \mathrm{~mol} \%$ styrene or $3: 1$ styrene/DOPC molar ratio), an isotropic resonance emerges at $\mathrm{CS}_{\text {iso }}=-1.0 \mathrm{ppm}$ in the ${ }^{31} \mathrm{P}$ wideline spectrum that reflects the onset of small phospholipid assemblies in coexistence with the remaining styrene-saturated bilayer phase. The non-bilayer phase contributes about $5.6 \%$ of the overall spectral intensity. The appearance of the additional mesophase marks the maximum amount of styrene that a DOPC membrane can accommodate and the nature of styrene-induced membrane instability that occurs between 67 and $75 \mathrm{~mol} \%$ styrene.

Membranes of saturated chains DMPC, which model the chains of LPS in bacterial outer membranes, have a more ordered bilayer interior compared to DOPC. Overall, in the presence of styrene at concentrations as high as $80 \mathrm{~mol} \%(4: 1$ styrene/DMPC molar ratio), we observe persistence of a stable bilayer phase even at the highest styrene concentration (Figure $3 \mathrm{~b})$. Curiously, although little change is seen in the effective CSA and Pake width up to $67 \mathrm{~mol} \%$, higher concentrations reveal some increase in membrane lipid disorder possibly accompanied by subtle bilayer thinning ( $c f$. Figure 8 ). This is also seen as a slight styrene concentration-dependent reduction in overall ${ }^{31} \mathrm{P}$ CSA (Figure $3 \mathrm{~b}$ ).

To understand how lipid chain composition affects membrane stability to styrene, we compared a set of membranes with identical PC headgroups and different chain compositions in the presence of $75 \mathrm{~mol} \%$ styrene near the limit of membrane stability (Figure 4). We compare unsaturated chain lipids, DEPC and DOPC, mixed-chain POPC, and a series of saturated 12-16 carbon chain lipids, DLPC, DMPC, and DPPC. Wideline ${ }^{31} \mathrm{P}$ NMR spectra were acquired above the respective lipid transition temperatures at $20{ }^{\circ} \mathrm{C}$ for DEPC, DOPC, POPC, and DLPC; $28{ }^{\circ} \mathrm{C}$ for DMPC; and $45{ }^{\circ} \mathrm{C}$ for DPPC to assess the potential onset of non-bilayer phases. In the absence of styrene, all hydrated lipids assembled into stable bilayers that gave rise to Pake distributions in the ${ }^{31} \mathrm{P}$ NMR spectra. In the presence of 75 mol \% styrene, all three saturated lipids of increasing chain length, DLPC (C12:0), DMPC (C14:0), and DPPC (C16:0), retained the bilayer phase while showing increase in membrane disorder seen as a minor reduction in effective ${ }^{31} \mathrm{P}$ CSA. A hint of non-bilayer structures was noticed for DPPC only. By contrast, membrane with unsaturated lipid chains showed somewhat lower tolerance to styrene. Asymmetric POPC (C16:0; C18:1 $\Delta$ 9-cis) behaved similarly to DPPC with almost imperceptible membrane disruption. Although DPPC, POPC, and DOPC have matching hydrophobic thickness, DOPC bilayers appear to accommodate lower amounts of styrene compared to POPC or DPPC. This is unlike our previous observation of higher tolerance for butyl methacrylate in unsaturated membranes ${ }^{31}$ and shows that cis-unsaturated chains present a serious obstacle to accommodating the bulky styrene ring in the membrane interior.

The observed styrene tolerance reduction in cis-unsaturated chain lipids can potentially be ameliorated in trans-unsaturated chain lipids. Indeed, such trans-unsaturated lipids, introduced into $E$. coli from recombinant $P$. putida cis-trans isomerase, have been shown to confer enhanced tolerance to organic solvents in growth cultures. ${ }^{44}$ To understand the impact of trans $v$ s cis chain unsaturation styrene tolerance, we assembled DEPC (C18:1 $\Delta$ 9-trans) membrane in the presence of $75 \mathrm{~mol} \%$ styrene. As a result of chain extension in the trans conformation, the hydrophobic thickness of DEPC matches that of the longer-chain saturated lipid DSPC (C18:0) that allows better chain packing within the membrane interior. Curiously, DEPC membranes showed similar level of bilayer phase disruption as DOPC in the presence of $75 \mathrm{~mol} \%$ styrene, observed as a sharp symmetrical peak at $-1 \mathrm{ppm}$, contributing to about $4.5 \%$ of the overall spectral intensity. This reveals an important role of increased mid-chain rigidity in styrene tolerance and points to styrene localization near the mid-chain depth, unlike the more interfacial location of buthyl methacrylate. In contrast to BMA, which has a carbonyl moiety resembling the lipid ester links, styrene is an aromatic hydrocarbon with affinity for the aliphatic membrane interior. 

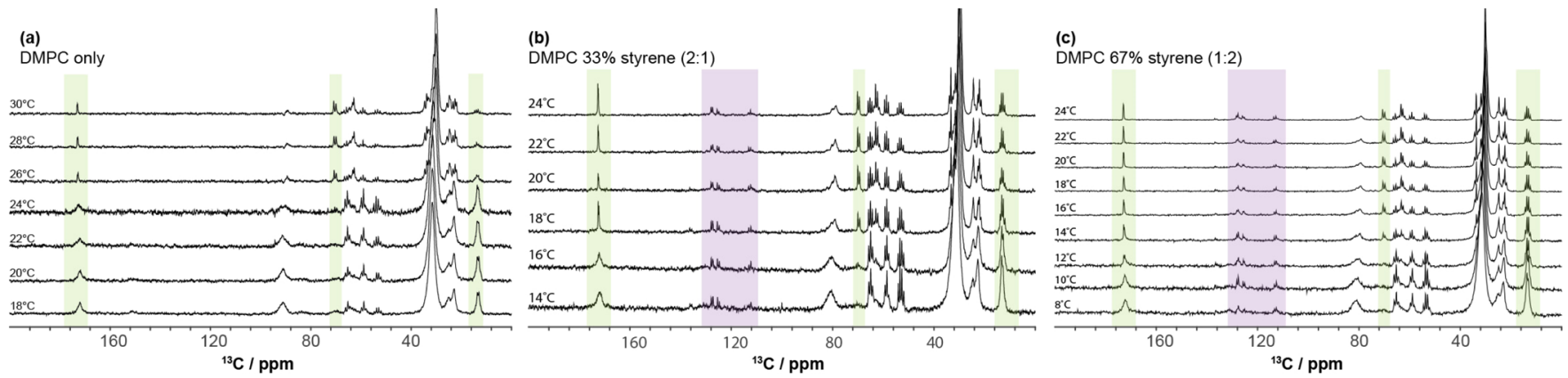

Figure 6. High-resolution J-resolved LG-CP ${ }^{13} \mathrm{C}$ MAS NMR spectra of hydrated DMPC MLVs with 0, 33 and 67 mol \% styrene. The transition temperature of DMPC is lowered between 16 and $18{ }^{\circ} \mathrm{C}$ in the presence of $33 \mathrm{~mol} \%$ styrene and further lowered between 10 and $14{ }^{\circ} \mathrm{C}$ in the presence of $67 \mathrm{~mol} \%$ styrene. Purple highlights styrene signals, and green highlights DMPC resonances used to determine the main transition temperature $T_{\mathrm{m}}$.

In contrast to styrene, on addition of hydrophobic polymer precursor butyl methacrylate, DEPC showed a better tolerance to BMA incorporation than DOPC, which reflects differences in packing and molecular localization of the apolar aromatic styrene molecule and the more amphipolar, linear BMA molecule. Changes to the Pake pattern width $(\sim 4 \mathrm{ppm})$ were observed for all lipid types used in this study, with the incorporation of $75 \mathrm{~mol} \%$ styrene leading to a decreased in Pake distribution. This effect appeared to be more prominent with DPPC, DMPC, and DLPC, suggesting that the changes in membrane dynamic were more drastic in saturated lipids compared to the unsaturated one, despite showing little to no lipid bilayer disruption. This is a direct reflection of the disordering effect the bulky aromatic ring of styrene has on lipid chain packing.

The proposed mechanism of styrene toxicity to membranes stems from the hydrophobic nature of its molecule $(\log P=$ $2.95)$, which readily partitions into the hydrophobic interior of membranes and accumulates at high levels causing chain/ headgroup area mismatch. On reaching a critical level, this leads to budding of lipid from the bilayer and the formation of non-bilayer aggregates and membrane collapse. To observe the accumulation of styrene into the membrane hydrophobic interior, we use high-resolution ${ }^{13} \mathrm{C}$ CP MAS NMR from natural abundance ${ }^{13} \mathrm{C}$ in styrene and membrane lipid. $\mathrm{CP}$ MAS NMR uniquely reports the motionally restricted molecular environments and can be used as a filter to distinguish membrane-incorporated styrene while concealing styrene in solution. Figure 5 shows ${ }^{13} \mathrm{C}$ CP MAS NMR spectra from fluid DEPC, DOPC, POPC, DPPC, DMPC, and DLPC membranes with $75 \mathrm{~mol} \%$ styrene recorded under $5 \mathrm{kHz}$ MAS above the respective lipid transition temperature. Chemical shift assignment of styrene and lipids is shown in Figure S1. The lipid ${ }^{13} \mathrm{C}$ CP MAS spectra show high-resolution features characteristic of liquid crystalline lipids bilayer above transition temperature. Curiously, despite its volatility, styrene remained membrane-embedded, and hydration was maintained over extended periods of time (30+ days) under $5 \mathrm{kHz}$ MAS (Figure S2).

Resonance intensity from membrane-embedded styrene can be quantified with good confidence against lipid intensity due to similarity of their molecular dynamics in the common membrane environment. The relative resonance intensities in Figure 5 show good quantitative agreement with molar ratios in each sample, and styrene titration into DMPC membranes shows clear increase in styrene intensity with increasing amounts of added styrene from 0 up to $75 \mathrm{~mol} \%$ (Figure S3). Styrene in membranes was also observed using ${ }^{1} \mathrm{H},{ }^{13} \mathrm{C}$ HETCOR (Figure S4), revealing good intramolecular correlations but little intermolecular magnetization exchange, indicative of high molecular mobility in the fluid membranes.

Chain interactions within the membrane hydrophobic core and trans-gauche mobility of lipid acyl chains are main determinants of lipid chain melting temperature, $T_{\mathrm{m}}$. The main transition temperature reflects the structure of the phospholipid acyl chains both in length - DLPC (C12:0, -2 $\left.{ }^{\circ} \mathrm{C}\right)$, DMPC $\left(14: 0,24{ }^{\circ} \mathrm{C}\right)$, and DPPC $\left(16: 0,42{ }^{\circ} \mathrm{C}\right)$ and in unsaturation with DOPC $\left(18: 1 \Delta 9\right.$-cis, $\left.-17{ }^{\circ} \mathrm{C}\right)$ and DEPC $\left(18: 1 \Delta 9\right.$-trans, $\left.12{ }^{\circ} \mathrm{C}\right)$. As such, the main transition temperature is a sensitive measure of changes in lipid packing and molecular interactions within the hydrophobic membrane interior.

Styrene incorporation into the membrane interior affects membrane packing, chain order, and the lipid main transition temperature. We use high-resolution J-resolved ${ }^{13} \mathrm{C}$ CP MAS NMR under Lee-Goldburg decoupling to monitor changes in lipid segmental dynamics that result from styrene incorporation into the membrane interior and the associated shift in main transition temperature. High-resolution ${ }^{13} \mathrm{C}$ CP MAS under FSLG decoupling scheme is able to provide $J$-resolve spectra sensitive to changes in lipid segmental dynamics, reporting lipid bilayer phases and transition temperature. Fluid membranes give rise to sharp and well-resolved proximal ${ }^{\mathrm{HC}} \mathrm{J}$ multiplet structures, as fast molecular rotation removes residual proton dipolar interactions. By contrast, membranes in the tilted lamellar gel phase, $\mathrm{P}_{\beta}$, have tighter molecular packing and reduced molecular mobility, which affect both $J$-multiplet resolution and resonance intensity under CP MAS. This offers a clear indication of the change in lipid phase state and reveals molecular segments and mixture components, involved in the transition.

Styrene incorporation into the membrane hydrophobic interior leads to uncoupling of thermally driven trans-gauche dynamics from collective chain-chain packing. Although the first effect is thermodynamically driven, the latter is modulated by styrene intercalation between lipid chains. To untangle the contribution from these two phenomena, we monitor the impact of styrene addition on the DMPC/styrene mixed membrane phase behavior. Following changes in their $J$ resolved ${ }^{13} \mathrm{C}$ LG-CP MAS NMR spectra with temperature, we compare hydrated DMPC membranes alone, DMPC with 33 mol \% styrene (2:1 lipid-to-styrene), and DMPC with $67 \mathrm{~mol}$ 
$\%$ styrene (1:2 lipid-to-styrene) (Figure 6). Hydrated pure DMPC membranes have $T_{\mathrm{m}}$ of $24{ }^{\circ} \mathrm{C}$ and, as seen in Figure $6 \mathrm{a}$, have a marked drop in resolution across the fluid to gel transition. At $30{ }^{\circ} \mathrm{C}$, above $T_{\mathrm{m}}$ of DMPC, J-resolved mutliplets are clearly observed. Quartets from the methyl carbons at 14 ppm (chain) and $54 \mathrm{ppm}$ (headgroup) are well resolved with proximal $J^{\mathrm{CH}}$ around 120 and $95 \mathrm{~Hz}$, respectively. The glycerol sn-2' gives rise to a distinct double at $70 \mathrm{ppm}$, with the $J^{\mathrm{CH}}$ of $124 \mathrm{~Hz}$. The J-coupling values determined from FSLGdecoupled ${ }^{13} \mathrm{C}$ MAS $\mathrm{CP}$ spectra from headgroup resonances are higher than in the acyl chains on the order of 155 and 120 $\mathrm{Hz}$ for methylene and methyl resonances, respectively. The actual values of $J^{\mathrm{CH}}$ are scaled by $\sqrt{3}$ from the $J^{\mathrm{CH} *}$ values observed under LG decoupling (Figure 6). ${ }^{36,37}$

The LG-CP measurements allow us to obtain proximal couplings from the $J$-resolved multiplets from styrene, such as ring carbons with resonances around 126-128 ppm and the methacrylate $\underline{\mathrm{CH}}$ doublets for S8 at 113 and $141 \mathrm{ppm}$. Styrene resonances can only be observed from membrane-incorporated styrene, and the normalized $J^{\mathrm{CH}}$ values of $126 \mathrm{~Hz}$ can be obtained from the LG-scaled couplings. An interesting observation from Figure $6 \mathrm{~b}, \mathrm{c}$ is that the spectral intensity from the styrene multiplets is enhanced in the gel phase, which reveals a shift from rapid, independent styrene dynamics within the fluid membrane to more cooperative dynamics following the reduction in lipid chain mobility. The binary phase diagram of DMPC/styrene, Figure 7, shows the effect of styrene

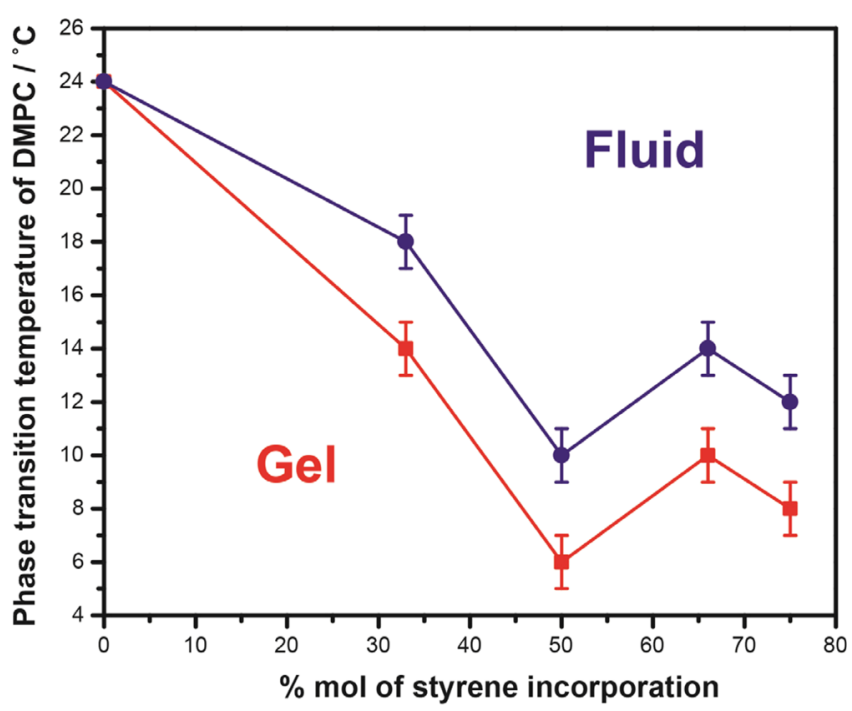

Figure 7. Outline of the hydrated DMPC/styrene phase diagram showing suppression of DMPC main chain melting transition temperature, $T_{\mathrm{m}}$, determined from the LG-CP ${ }^{13} \mathrm{C}$ MAS NMR. The liquidus between the liquid crystal, $\mathrm{L}_{\alpha}$, phase and the two phase coexistence region is outlined in blue, and the solidus between the two phase coexistence and the tilted gel phase, $\mathbf{L}_{\beta}$, is shown in red. ${ }^{52}$ The eutectic depression in transition temperature near 1:1 styrene/lipid ratio was consistent over three samples and NMR repeats and reveals an optimal packing of styrene ring in the hydrophobic lipid interior.

incorporation on DMPC phase transition temperature, determined from ${ }^{13} \mathrm{C}$ CP NMR with FSLG decoupling. The general trend of decrease in DMPC $T_{\mathrm{m}}$ with increasing concentration of styrene can be clearly observed, suggesting that styrene molecule intercalates and immobilizes between the lipid acyl chains. The incorporation of styrene leads to disruption of lipid membrane packing order, whereas the lipid bilayer structure is preserved as suggested by ${ }^{31} \mathrm{P}$ wideline experiment shown in Figure 3b. Curiously, at $50 \mathrm{~mol} \%$ styrene (1:1 molar ratio), we observe a dip in the transition temperature and deviation from ideal mixing behavior. As both solidus and liquidus are suppressed, rather than merged, this is not a eutectic point but the result of particularly perturbing styrene packing at this ratio within the membrane hydrophobic interior.

Solid state NMR data indicate a reduction in the thickness of saturated lipid membranes at styrene concentrations above 67 mol \%. We use SAXS to monitor the effect of styrene incorporation on membrane thickness in multilamellar lipid suspensions on different chain compositions. Hydrated MLV suspensions of two unsaturated, DOPC (C18:1 $\Delta$ 9-cis), DEPC (C18:1 4 -trans) and two saturated PCs, DMPC (C14:0) and DPPC (C16:0), were studied in the presence of increasing concentrations of styrene (Figure 8 ). The scattering profiles from all lipids were dominated by strong Bragg reflections with integer spacing clearly visible for $n=1$ and 2 with the presence of third-order Bragg reflection in the unsaturated systems, a clear indication of a bilayer phase with periodic lamellar repeat at all styrene concentrations. Bilayer repeat was maintained with subtle variation in the presence of styrene-minor decrease in thickness for saturated lipids and subtle increase in DOPC; no change was observed in DEPC bilayer (Figure S5).

Lipid chains vary in effective hydrophobic length from 14 (DMPC) through 16 (DPPC, DOPC) to 18 carbons (DEPC), and first-order reflections were observed between $q=0.094$ and $0.101 \AA^{-1}(d=68-62 \AA)$ for the limiting cases of DEPC and DMPC, respectively. In the presence of increasing concentration of styrene: $33 \mathrm{~mol} \%$ (2:1 lipid-to-styrene), 50 mol \% (1:1 lipid-to-styrene), $67 \mathrm{~mol} \%$ (1:2 lipid-to-styrene), $75 \mathrm{~mol} \%$ (1:3 lipid-to-styrene), and $80 \mathrm{~mol} \%$ (1:4 lipid-tostyrene), no further reflections were observed and the main Bragg reflections from each lipid membrane were observed roughly at unchanged $q$-values. On closer inspection, the firstorder Bragg reflections showed some small variation with styrene concentration (Figure S5).

The cis-unsaturated lipid, DOPC, has an 18-carbon chain with effective hydrophobic projection equivalent to a 16carbon saturated (palmitic) chain and gives rise to $n=1$ reflection at $q=0.1004 \AA^{-1}$ in the absence of any styrene. The addition of styrene leads to a small drop in $q=0.0999 \AA^{-1}$ at $50 \mathrm{~mol} \%$ and further to $q=0.0996 \AA^{-1}$ with $80 \mathrm{~mol} \%$ styrene. The decrease in $q$ value reveals a small initial increase in lamellar repeat from 62.2 to $63.1 \AA$, which changes little on further increase in styrene concentration.

Saturated DMPC showed the opposite trend with $n=1$ reflection shifting from $q=0.0995 \AA^{-1}$ in the absence of styrene to $q=0.1002 \AA^{-1}$ for DMPC with $50 \mathrm{~mol} \%$ styrene that remained almost unchanged at $q=0.1005 \AA^{-1}$ for $80 \mathrm{~mol}$ $\%$ styrene, revealing a small initial decrease in lamellar repeat from 63.1 to $62.5 \AA$ on styrene addition.

The longer-chain saturated lipid, DPPC with 16-carbon chains, matching the hydrophobic thickness of DOPC, displayed similar response to styrene as DMPC. First-order reflections for pure lipid were observed at $q=0.0938 \AA^{-1}$ and shifted to $q=0.0949 \AA^{-1}$ for DPPC with $80 \mathrm{~mol} \%$ styrene. The increase in $q$ value indicates that the lamellar repeat decreased from 67.0 to $66.2 \AA$ with high styrene content. Again, the change in lamellar repeat is most pronounced on initial styrene addition, and its subsequent increase in 

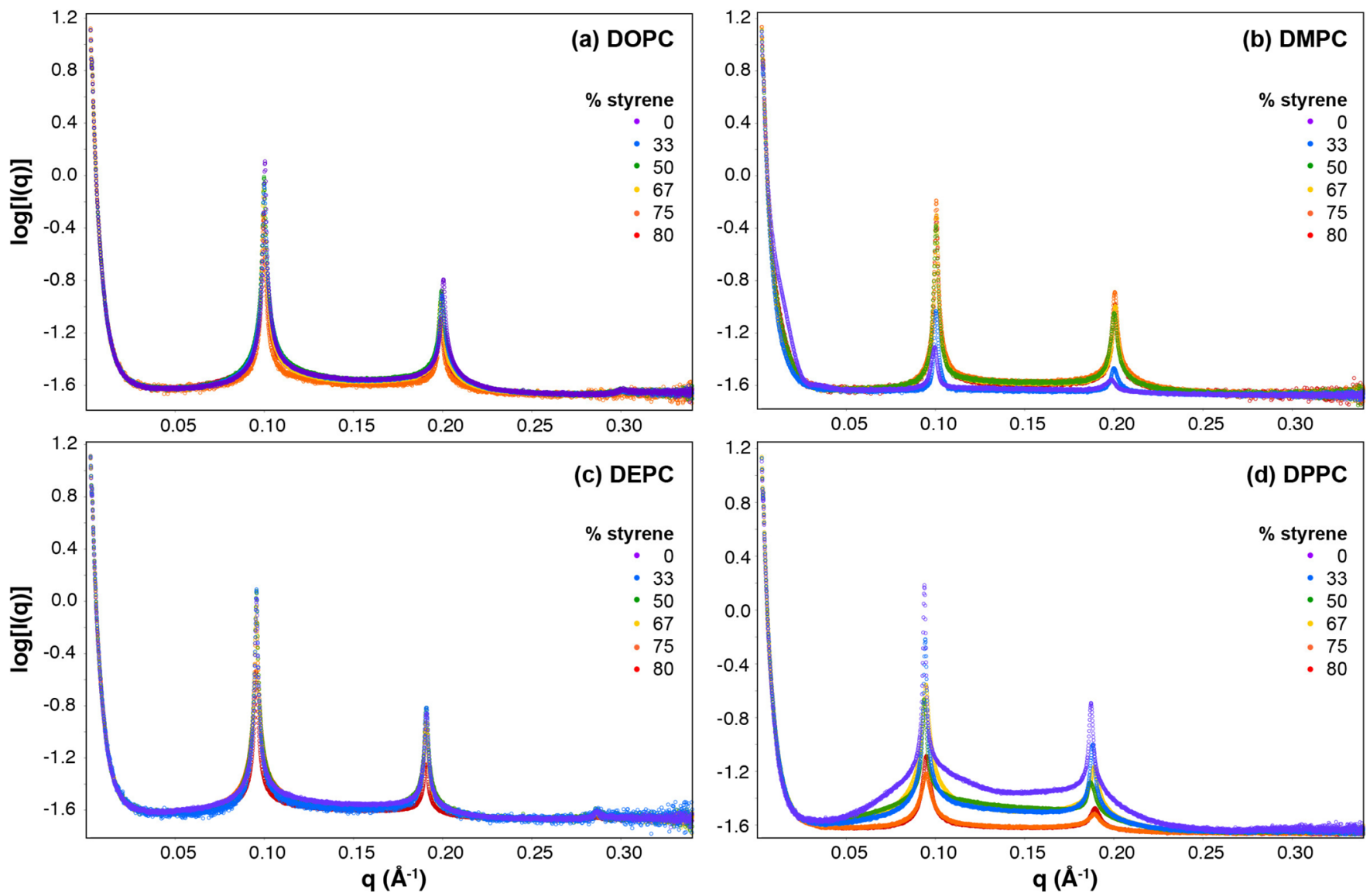

Figure 8. Small angle X-ray scattering 1D profiles from DOPC, DMPC, DEPC, and DPPC membranes in MLV structures recorded at $20{ }^{\circ} \mathrm{C}$ for DOPC and DEPC, $30^{\circ} \mathrm{C}$ for DMPC, and $45{ }^{\circ} \mathrm{C}$ for DPPC and in the presence of styrene at $0,33,50,67,75$, and 80 mol \% in hydrated (a) DMPC, (b) DOPC, (c) DEPC, and (d) DPPC MLVs. Bragg reflections at $n=1,2$ and a hint of $n=3$ confirm the presence of a bilayer phase with lamellar repeat between $d=68$ and $62 \AA$.

concentration has little effect. In all three lipids, changes in bilayer repeat occurred largely on first introduction of styrene with very small concentration-dependent variation above 33 mol \%. This differs from the NMR observations where changes in membrane dynamics and order became pronounced above $67 \mathrm{~mol} \%$ styrene and reveals two stages in styrene incorporation-initial rearrangement of bilayer hydrophobic interior, seen as change in lamellar repeat below $33 \mathrm{~mol} \%$ styrene, followed by lateral swelling that increases headgroup mobility, detected as ${ }^{31} \mathrm{P}$ effective CSA decrease only above 67 mol \% styrene.

Like DOPC, DEPC has symmetric 18-carbon chains but with trans unsaturation in the middle of the chain, which causes markedly reduced perturbation to chain order and a greater chain extension equivalent to saturated 18-carbon chains. Although styrene incorporates into the bilayer, as seen in the ${ }^{13} \mathrm{C}$ NMR spectra, unlike the DOPC, DMPC, and DPPC membranes, there appears to be no perceptible change in bilayer repeat with $q=0.0957 \AA^{-1}$ for DEPC alone and $q=$ $0.0955 \AA^{-1}$ with $80 \mathrm{~mol} \%$ styrene $(d=65.5$ and $65.8 \AA$, respectively). This is consistent with the ${ }^{31} \mathrm{P}$ NMR data and shows that styrene incorporates very well into the DEPC membranes without causing noticeable changes in membrane structure and organization, which is likely the result of the chain extension at position 9. Curiously, membrane collapse occurs in DEPC, as well as in DOPC above $67 \mathrm{~mol} \%$ styrene, which reveals a hard "ceiling" in the capacity of these membranes to accommodate styrene, which correlates with the presence of the hard "plate"-like double bond at mid-chain that clashes with the styrene ring packing. This is exacerbated by preferred co-localization of styrene in the vicinity of the lipid chain double bond, which is mediated by $\pi$ - $\pi$ interactions with delocalized electron density in the styrene ring.

The absence of any non-integer reflections at high styrene levels in the unsaturated lipid mixtures reveals that the isotropic intensity, observed in the wideline NMR spectra of DOPC and DEPC above $67 \mathrm{~mol} \%$ styrene, arises from small, rapidly tumbling non-bilayer aggregates and is not the result of lamellar to cubic phase conversion. The approximate size of such non-bilayer assemblies falls outside the observed $q$-range and requires separate analysis.

The possibility exists that at high styrene concentrations a styrene "pool" may form between the two bilayer leaflets, which can uncouple from each other. This would be seen as a marked increase in bilayer repeat, which we do not observe in any of the investigated lipid systems. This is in line with previous studies of membrane saturation with butyl methacrylate $^{31}$ and the corresponding solid state NMR data. However, in contrast to BMA, which localizes in close proximity to the lipid carbonyls, styrene is entirely apolar and most likely entirely immersed into the membrane hydrophobic interior. The $14{ }^{\circ} \mathrm{C}$ depression in chain melting temperature, seen in the ${ }^{13} \mathrm{C}$ NMR data, supports the model of styrene incorporation into the membrane lipid core. This 
would place the bulky styrene ring in direct conflict with the structured double bond of unsaturated lipids and explains the reduced ability of DOPC and DEPC bilayers to accommodate styrene in comparison to saturated DMPC and DPPC, observed by ${ }^{31} \mathrm{P}$ NMR.

\section{CONCLUSIONS}

Increasing demand for biorenewable production of styrene and other commodity chemicals drives the development of largescale biofermentative manufacturing technologies, where solvent toxicity to host organism remains a technological bottleneck. Membrane instability at production levels of solvent is a limiting factor, which can be countered by engineering bacterial production strains with enhanced membrane stability and solvent export systems. Here, we investigate the role of lipid chain composition on membrane stability at high levels of styrene. Saturated chain lipids, DMPC and DPPC, were found to retain bilayer phase stability to markedly higher levels of styrene than unsaturated lipids DOPC and DEPC. Although gradually yielding to non-bilayer phase conversion above $67 \mathrm{~mol} \%$ styrene, trans-unsaturated 18-carbon chain DEPC membrane showed remarkably consistent order and dynamics and membrane properties virtually unaffected by styrene up to $80 \mathrm{~mol} \%$, which reveals their superior ability to support the structure and function of membrane proteins and pumps.

Saturated lipid membranes accommodate styrene better than unsaturated membranes, showing little to no signs of bilayer phase disruption at concentration of styrene as high as $80 \mathrm{~mol} \%$. Longer saturated chain membranes, DPPC (16carbon), appear slightly more affected by styrene than DMPC (14-carbon) and DLPC (12-carbon), suggesting higher tolerance in the less ordered, shorter lipid chains. This trend is extended to include the reduced tolerance in longer-chain trans-unsaturated DEPC (C18:1 $\Delta$ 9-trans) with 18-carbon effective hydrophobic length. Chain unsaturation, however, appears to have a dominantly detrimental impact on membrane stability that manifests for both cis- (DOPC) and trans- (DEPC) isomers. Interestingly, despite its volatility, all membranes readily accepted and retained styrene even under harsh mechanical spinning and ambient temperature exposure over extended periods of time.

Bacterial survival in the presence of short-chain hydrocarbons includes adaptive reduction in membrane lipid chain length. Based on our observations, we propose a model, in which the underlying adaptive chain length reduction in bacteria is not driven by any metabolic or transcriptional cascade but is a fitness-driven selection, in which accumulation of shorter-chain lipids confers survival advantage through enhanced membrane stability. In addition, conversion of cis- to trans-unsaturation through metabolic engineering, such as the introduction into $E$. coli of cis/trans isomerase from $P$. aeruginosa, ${ }^{44}$ can also enhance membrane stability to styrene.

\section{ASSOCIATED CONTENT}

\section{SI Supporting Information}

The Supporting Information is available free of charge at https://pubs.acs.org/doi/10.1021/acs.langmuir.1c02332.

Table of lipid-to-styrene ratios and corresponding mol $\%$, additional ${ }^{13} \mathrm{C}$ MAS CP NMR spectra of DMPC with different amount of styrene, heteronuclear correlations of hydrated DMPC with styrene, and additional SAXS scattering profile focusing on the first-order reflections (PDF)

\section{AUTHOR INFORMATION}

\section{Corresponding Author}

Boyan B. Bonev - School of Life Sciences, University of Nottingham, Nottingham NG7 2UH, U.K.; orcid.org/ 0000-0001-7156-2412; Email: boyan.bonev@

nottingham.ac.uk

\section{Authors}

Vivien Yeh - School of Life Sciences, University of Nottingham, Nottingham NG7 2UH, U.K.; ๑ orcid.org/ 0000-0003-2232-2329

Alice Goode - School of Life Sciences, University of Nottingham, Nottingham NG7 2UH, U.K.

David Johnson - Lucite International, Wilton Centre, Redcar TS10 4RF, U.K.

Nathan Cowieson - Diamond Light Source, Oxford OX22 ODE, U.K.

Complete contact information is available at:

https://pubs.acs.org/10.1021/acs.langmuir.1c02332

\section{Notes}

The authors declare no competing financial interest.

\section{ACKNOWLEDGMENTS}

This work was supported by the Biotechnology and Biological Sciences Research Council BB/N010426/1 to B.B.B. We would like to thank Diamond Light Source for access to B21 beamline (SM16125 and SM21035-195). B21 beam-time on experimental line was granted by Diamond via rapid access route (http://www.diamond.ac.uk/Users.html). Solid state NMR instrument funding was provided by the Biotechnology and Biological Sciences Research Council (grant BB/ C510924) to BBB. BB would also like to thank the NVIDIA Corporation for the GPGPU hardware grant.

\section{REFERENCES}

(1) Wu, C.-Y.; Kobylinski, T. P.; Bozik, J. E. Preparation of Styrene from Ethylbenzene. US4255599A, March 10, 1981.

(2) Van Dien, S. From the First Drop to the First Truckload: Commercialization of Microbial Processes for Renewable Chemicals. Curr. Opin. Biotechnol. 2013, 24, 1061-1068.

(3) Tang, W. L.; Zhao, H. Industrial Biotechnology: Tools and Applications. Biotechnol. J. 2009, 4, 1725-1739.

(4) Pagot, Y.; Belin, J.-M.; Husson, F.; Spinnler, H.-E. Metabolism of Phenylalanine and Biosynthesis of Styrene in Penicillium Camemberti. J. Dairy Res. 2007, 74, 180-185.

(5) Fernandez, X.; Lizzani-Cuvelier, L.; Loiseau, A.-M.; Perichet, C.; Delbecque, C.; Arnaudo, J.-F. Chemical Composition of the Essential Oils from Turkish and Honduras Styrax. Flavour Fragr. J. 2005, 20, 70-73.

(6) McKenna, R.; Nielsen, D. R. Styrene biosynthesis from glucose by engineered E. coli. Metab. Eng. 2011, 13, 544-554.

(7) McKenna, R.; Moya, L.; McDaniel, M.; Nielsen, D. R. Comparing in Situ Removal Strategies for Improving Styrene Bioproduction. Bioprocess Biosyst. Eng. 2015, 38, 165-174.

(8) Lian, J.; McKenna, R.; Rover, M. R.; Nielsen, D. R.; Wen, Z.; Jarboe, L. R. Production of Biorenewable Styrene: Utilization of Biomass-Derived Sugars and Insights into Toxicity. J. Ind. Microbiol. Biotechnol. 2016, 43, 595-604.

(9) Chen, C.; Ding, S.; Wang, D.; Li, Z.; Ye, Q. Simultaneous Saccharification and Fermentation of Cassava to Succinic Acid by Escherichia Coli NZN111. Bioresour. Technol. 2014, 163, 100-105. 
(10) Becker, J.; Lange, A.; Fabarius, J.; Wittmann, C. Top Value Platform Chemicals: Bio-Based Production of Organic Acids. Curr. Opin. Biotechnol. 2015, 36, 168-175.

(11) Qi, Y.; Liu, H.; Chen, X.; Liu, L. Engineering Microbial Membranes to Increase Stress Tolerance of Industrial Strains. Metab. Eng. 2019, 53, 24-34.

(12) Morein, S.; Andersson, A.-S.; Rilfors, L.; Lindblom, G. WildType Escherichia Coli Cells Regulate the Membrane Lipid Composition in a Window between Gel and Non-Lamellar Structures. J. Biol. Chem. 1996, 271, 6801-6809.

(13) Kaneda, T. Iso- and Anteiso-Fatty Acids in Bacteria: Biosynthesis, Function, and Taxonomic Significance. Microbiol. Mol. Biol. Rev. 1991, 55, 288-302.

(14) Weber, F. J.; de Bont, J. A. M. Adaptation Mechanisms of Microorganisms to the Toxic Effects of Organic Solvents on Membranes. Biochim. Biophys. Acta 1996, 1286, 225-245.

(15) Buttke, T. M.; Ingram, L. O. Mechanism of Ethanol-Induced Changes in Lipid Composition of Escherichia Coli: Inhibition of Saturated Fatty Acid Synthesis in Vivo. Biochemistry 1978, 17, 637644.

(16) Dombek, K. M.; Ingram, L. O. Effects of Ethanol on the Escherichia Coli Plasma Membrane. J. Bacteriol. 1984, 157, 233-239.

(17) Heipieper, H. J.; Weber, F. J.; Sikkema, J.; Keweloh, H.; de Bont, J. A. M. Mechanisms of Resistance of Whole Cells to Toxic Organic Solvents. Trends Biotechnol. 1994, 12, 409-415.

(18) Heipieper, H. J.; de Bont, J. A. Adaptation of Pseudomonas Putida S12 to Ethanol and Toluene at the Level of Fatty Acid Composition of Membranes. Appl. Environ. Microbiol. 1994, 60, $4440-4444$

(19) Ramos, J. L.; Duque, E.; Rodríguez-Herva, J.-J.; Godoy, P.; Haïdour, A.; Reyes, F.; Fernández-Barrero, A. Mechanisms for Solvent Tolerance in Bacteria. J. Biol. Chem. 1997, 272, 3887-3890.

(20) Ingram, L. O. Adaptation of Membrane Lipids to Alcohols. J. Bacteriol. 1976, 125, 670-678.

(21) Mishra, P.; Prasad, R. Role of Phospholipid Head Groups in Ethanol Tolerance of Saccharomyces Cerevisiae. Microbiology 1988, 134, 3205-3211.

(22) Pinkart, H. C.; Wolfram, J. W.; Rogers, R.; White, D. C. Cell Envelope Changes in Solvent-Tolerant and Solvent-Sensitive Pseudomonas Putida Strains Following Exposure to o-Xylene. Appl. Environ. Microbiol. 1996, 62, 1129-1132.

(23) Eliasz, A. W.; Chapman, D.; Ewing, D. F. Phospholipid Phase Transitions. Effects of n-Alcohols, n-Monocarboxylic Acids, Phenylalkyl Alcohols and Quatenary Ammonium Compounds. Biochim. Biophys. Acta 1976, 448, 220-233.

(24) Sardessai, Y.; Bhosle, S. Tolerance of Bacteria to Organic Solvents. Res. Microbiol. 2002, 153, 263-268.

(25) De Smet, M. J.; Kingma, J.; Witholt, B. The Effect of Toluene on the Structure and Permeability of the Outer and Cytoplasmic Membranes of Escherichia Coli. Biochim. Biophys. Acta 1978, 506, 64-80.

(26) Westerman, P. W.; Pope, J. M.; Phonphok, N.; Doane, J. W.; Dubro, D. W. The Interaction of N-Alkanols with Lipid Bilayer Membranes: A 2H-NMR Study. Biochim. Biophys. Acta 1988, 939, 64-78.

(27) Sullivan, K. H.; Hegeman, G. D.; Cordes, E. H. Alteration of the Fatty Acid Composition of Escherichia Coli by Growth in the Presence of Normal Alcohols. J. Bacteriol. 1979, 138, 133.

(28) Huffer, S.; Clark, M. E.; Ning, J. C.; Blanch, H. W.; Clark, D. S. Role of Alcohols in Growth, Lipid Composition, and Membrane Fluidity of Yeasts, Bacteria, and Archaea. Appl. Environ. Microbiol. 2011, 77, 6400-6408.

(29) Baez, A.; Cho, K.-M.; Liao, J. C. High-Flux Isobutanol Production Using Engineered Escherichia Coli: A Bioreactor Study with in Situ Product Removal. Appl. Microbiol. Biotechnol. 2011, 90, $1681-1690$

(30) Konas, R. M.; Daristotle, J. L.; Harbor, N. B.; Klauda, J. B. Biophysical Changes of Lipid Membranes in the Presence of Ethanol at Varying Concentrations. J. Phys. Chem. B 2015, 119, 13134-13141.
(31) Yeh, V.; Goode, A.; Eastham, G.; Rambo, R. P.; Inoue, K.; Doutch, J.; Bonev, B. B. Membrane Stability in the Presence of Methacrylate Esters. Langmuir 2020, 36, 9649-9657.

(32) Bonev, B.; Watts, A.; Bokvist, M.; Gröbner, G. Electrostatic Peptide-Lipid Interactions of Amyloid- $\beta$ Peptide and Pentalysine with Membrane Surfaces Monitored by 31P MAS NMR. Phys. Chem. Chem. Phys. 2001, 3, 2904-2910.

(33) Bonev, B. B.; Lam, Y.-H.; Anderluh, G.; Watts, A.; Norton, R. S.; Separovic, F. Effects of the Eukaryotic Pore-Forming Cytolysin Equinatoxin II on Lipid Membranes and the Role of Sphingomyelin. Biophys. J. 2003, 84, 2382-2392.

(34) Nagle, J. F.; Tristram-Nagle, S. Structure of Lipid Bilayers. Biochim. Biophys. Acta 2000, 1469, 159-195.

(35) Lane, W. H. Determination of Solubility of Styrene in Water and of Water in Styrene. Ind. Eng. Chem., Anal. Ed. 1946, 18, 295296.

(36) Ciesielski, F.; Griffin, D. C.; Rittig, M.; Bonev, B. B. HighResolution J-Coupled 13C MAS NMR Spectroscopy of Lipid Membranes. Chem. Phys. Lipids 2009, 161, 77-85.

(37) Zorin, V.; Ciesielski, F.; Griffin, D. C.; Rittig, M.; Bonev, B. B. Heteronuclear Chemical Shift Correlation and J-Resolved MAS NMR Spectroscopy of Lipid Membranes. Magn. Reson. Chem. 2010, 48, 925-934.

(38) Fung, B. M.; Khitrin, A. K.; Ermolaev, K. An Improved Broadband Decoupling Sequence for Liquid Crystals and Solids. J. Magn. Reson. 2000, 142, 97-101.

(39) Lee, M.; Goldburg, W. I. Nuclear-Magnetic-Resonance Line Narrowing by a Rotating Rf Field. Phys. Rev. 1965, 140, A1261.

(40) Brooks, B. R.; Bruccoleri, R. E.; Olafson, B. D.; States, D. J.; Swaminathan, S.; Karplus, M. CHARMM: A Program for Macromolecular Energy, Minimization, and Dynamics Calculations. J. Comput. Chem. 1983, 4, 187-217.

(41) Phillips, J. C.; Braun, R.; Wang, W.; Gumbart, J.; Tajkhorshid, E.; Villa, E.; Chipot, C.; Skeel, R. D.; Kalé, L.; Schulten, K. Scalable Molecular Dynamics with NAMD. J. Comput. Chem. 2005, 26, 17811802.

(42) Jo, S.; Kim, T.; Iyer, V. G.; Im, W. CHARMM-GUI: A WebBased Graphical User Interface for CHARMM. J. Comput. Chem. 2008, 29, 1859-1865.

(43) Pettersen, E. F.; Goddard, T. D.; Huang, C. C.; Couch, G. S.; Greenblatt, D. M.; Meng, E. C.; Ferrin, T. E. UCSF Chimera-A Visualization System for Exploratory Research and Analysis. J. Comput. Chem. 2004, 25, 1605-1612.

(44) Tan, Z.; Yoon, J. M.; Nielsen, D. R.; Shanks, J. V.; Jarboe, L. R. Membrane Engineering via Trans Unsaturated Fatty Acids Production Improves Escherichia Coli Robustness and Production of Biorenewables. Metab. Eng. 2016, 35, 105-113.

(45) Pake, G. E. Nuclear Resonance Absorption in Hydrated Crystals: Fine Structure of the Proton Line. J. Chem. Phys. 1948, 16, 327-336.

(46) Malcolm, I. C.; Ross, J. C.; Higinbotham, J. A Study of the Headgroup Motion of Sphingomyelin Using 31P NMR and an Analytically Soluble Model. Solid State Nucl. Magn. Reson. 2005, 27, 247-256.

(47) Brown, M. F.; Seelig, J. Influence of Cholesterol on the Polar Region of Phosphatidylcholine and Phosphatidylethanolamine Bilayers. Biochemistry 1978, 17, 381-384.

(48) Griffin, R. G. Observation of the Effect of Water on the Phosphorus-31 Nuclear Magnetic Resonance Spectra of Dipalmitoyllecithin. J. Am. Chem. Soc. 1976, 98, 851-853.

(49) Moreau, C.; Le Floch, M.; Segalen, J.; Leray, G.; Metzinger, L.; de Certaines, J. D.; Le Rumeur, E. Static and Magic Angle Spinning ${ }^{31}$ P NMR Spectroscopy of Two Natural Plasma Membranes. FEBS Lett. 1999, 461, 258-262.

(50) Ulrich, A. S.; Sami, M.; Watts, A. Hydration of DOPC Bilayers by Differential Scanning Calorimetry. Biochim. Biophys. Acta 1994, 1191, 225-230.

(51) Koynova, R.; Caffrey, M. Phases and Phase Transitions of the Phosphatidylcholines. Biochim. Biophys. Acta 1998, 1376, 91-145. 
(52) Bonev, B. B.; Morrow, M. R. Effect of Pressure on the Dimyristoylphosphatidylcholine Bilayer Main Transition. Phys. Rev. E 1997, 55, 5825-5833.

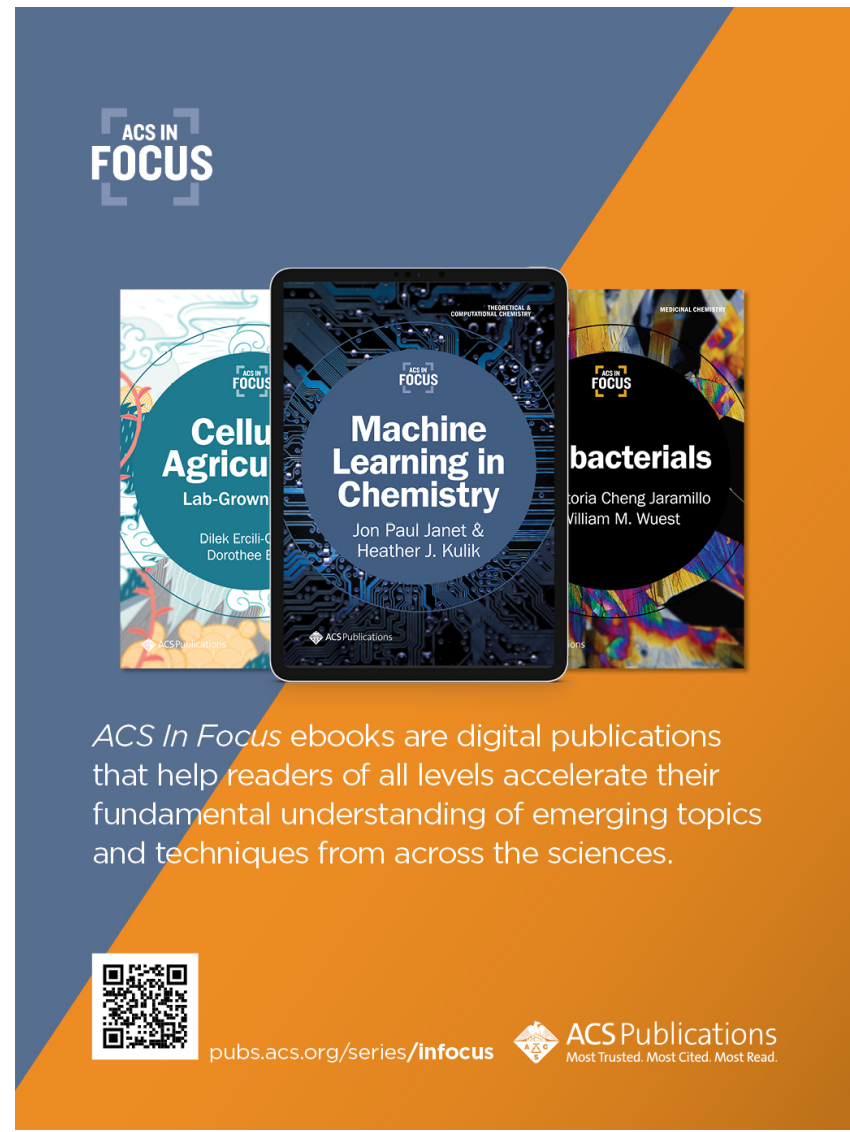

